\title{
Creep failure in a threshold-activated dynamics: Role of temperature during a subcritical loading
}

\author{
Subhadeep Roy* \\ PoreLab, Department of Physics, Norwegian University of Science and Technology, NO-7491 Trondheim, Norway \\ Takahiro Hatano $\oplus^{\dagger}$ \\ Department of Earth and Space Science, Osaka University, 560-0043 Osaka, Japan
}

(Received 11 November 2019; accepted 28 March 2020; published 29 April 2020)

\begin{abstract}
Creep is a time-dependent deformation of solids at relatively low stresses, leading to the breakdown with time. Here we propose a simple model for creep failure of disordered solids in which temperature and stress are controllable. Despite its simplicity, this model can reproduce most experimental observations: Distribution of the creep lifetime obeys the log-normal distribution, and the average creep lifetime decays in a scale-free manner with the increasing stress. Finally, to characterize the failure abruptness, the average breakdown frequency is introduced as an order parameter, which exhibits a series of jumps with varying temperature. Finite-size scaling holds for these jumps, implying the existence of nonequilibrium transitions.
\end{abstract}

DOI: 10.1103/PhysRevResearch.2.023104

\section{INTRODUCTION}

Failure point and the failure processes of disordered solids and composite materials depend on temperature, pressure, and driving conditions such as applied stress or strain rate [1]. Importantly, materials can deform and break with time, even if the applied stress is below the critical value. This phenomenon is known as creep failure or creep rupture. Study of creep failure is essential in many contexts such as constructions, in which the building blocks are subject to the load for a considerable duration. The time elapsed until creep failure is referred to as the creep lifetime. From a practical point of view, estimate of creep lifetime leads to a forecast of the catastrophic failure event and is therefore important in materials science. The creep lifetime depends on many ingredients, among which applied stress and temperature have prominent effects [2].

Since the physical mechanisms behind creep may be the accumulation of plastic strain and damage, models for creep should take such aging processes into account. Structural randomness and heterogeneity should be also considered appropriately. However, the fracture of heterogeneous solids is hard to handle with the elasticity theory, and therefore there have been some alternative approaches. A pioneering work by Main [3] is based on the subcritical crack growth dynamics and the phenomenological Voight's model for precursory strain [4]. Assuming a heterogeneous feedback system, the

\footnotetext{
*subhadeep.roy@ntnu.no

†hatano@ess.sci.osaka-u.ac.jp

Published by the American Physical Society under the terms of the Creative Commons Attribution 4.0 International license. Further distribution of this work must maintain attribution to the author(s) and the published article's title, journal citation, and DOI.
}

model can reproduce the time-dependent strain rate observed in experiments.

More recently, another approach has obtained popularity, making use of a simple and intuitive model for disordered solids known as the fiber bundle model (FBM) [5]. The original model is purely mechanical [6,7] and does not contain any effect of temperature. Nevertheless, the model can exhibit creeplike behaviors by interpreting the stress redistribution time step as the real time $[8,9]$. However, due to the absence of thermal effects, these creeplike behaviors can be observed only in the proximity of the critical stress. Thus, if we wish to propose a model for creep, thermal effects should be incorporated appropriately.

In the literature of FBM, the effect of temperature has been modeled in three different manners: (i) the applied stress fluctuates, (ii) fibers have plasticity, and (iii) the failure criterion for a single fiber is probabilistic. The first approach is quite simple but appears to be successful in explaining the time-dependent strain rate as well as the temperature and the stress (or strain) dependencies in the creep lifetime [10-14]. The fluctuating stress approach is also applicable to some other purposes $[15,16]$. In the second approach, fibers are not completely elastic but possess complex rheological properties. For instance, the time evolution equation for the strain of each fiber is introduced based on the Kelvin-Voigt rheology [17,18] or a nonlocal rheology [19]. The model behavior may somewhat depend on the specific rheology [20]. One can also consider damage rheology for each fiber [21]. These models may be categorized into the second approach, and they can reproduce time-dependent strain rate as well as the temperature and the stress dependences of creep lifetime. Naturally, the results may depend on the specific assumptions on the rheological properties of fibers.

The third approach, in which the failure rate of fibers depends on temperature, may be less common but possesses remarkable simplicity. The present study is classified into 
this category. In the literature, we are aware of two relevant studies [22,23]. They adopt as the failure rate some probability functions that are similar to the Arrhenius factor, but the creep behaviors are not thoroughly investigated. Here we adopt a similar yet simpler function for the failure rate, thus bringing thermal fluctuations into the model in addition to the timeindependent (i.e., quenched) randomness. Interplay between the two kinds of randomness yields nontrivial time-dependent behaviors. We have numerically studied the model focusing on the effects of temperature and applied stress on the creep behaviors: statistics of the creep lifetime and the avalanche statistics. In the next section, we provide a detailed description of the conventional fiber bundle model along with the modifications we have made for the present work. This is followed by the numerical results together with some brief comments and discussions on the future scope as a continuation of the present observations.

\section{DESCRIPTION OF THE MODEL}

The model consists of $L$ vertical fibers in between two parallel bars. A load $F$ is applied to the bars to create a stress $\sigma=F / L$ per fiber. Each fiber has an individual strength chosen from a threshold distribution randomly. This heterogeneity in the strength may be regarded as a quenched randomness in the model, and the dispersion of threshold distribution measures the extent of disorder in the model. When the applied stress exceeds a threshold value, the corresponding fiber breaks irreversibly and the load borne by that fiber is redistributed within the model, either among all the surviving fibers (mean-field model) or among the surviving nearest neighbors only (local stress concentration). Due to such redistribution, the local stress values of some fibers increase, and that can lead to further breaking and redistribution. This is an avalanche. After a certain number of avalanches, the model breaks completely or relaxes to a stable state with nonzero surviving fibers. In the latter case, the external load $F$ needs to increase to break the next weakest fiber, leading to further avalanches. This process continues until all the fibers are broken. The applied load just before the global failure is referred to as the critical load $F_{c}$.

Here we adopt the mean-field model for stress redistribution. To incorporate the effect of temperature in creep, we introduce a probabilistic failure criterion for a single fiber, namely, the probability of the $i$ th fiber to break at time $t$ is given by

$$
P_{r}(t, i)=P^{\prime} \exp \left[\frac{\sigma(t, i)-\sigma_{\mathrm{th}}(i)}{T}\right] .
$$

Here $\sigma(t, i)$ and $\sigma_{\text {th }}(i)$ are, respectively, the local stress and the stress threshold of the $i$ th fiber at time $t$, and $P^{\prime}$ is a constant chosen to be unity hereafter. With this criterion, any fibers can rupture even if the local stress is less than its threshold value. If $\sigma(t, i) \geqslant \sigma_{\mathrm{th}}(i), P_{r}$ reaches unity and the fibers break with probability 1 . Note that Eq. (1) is just an Arrhenius factor and is similar to those adopted previously [22,23], but yet it is slightly different. This model reduces to a conventional one [8] in the limit of $T \rightarrow 0$, in which a fiber can break only when $\sigma(i) \geqslant \sigma_{\text {th }}(i)$.
The time evolution of the system is as follows. At $t=0$, the load is applied to the system, leading to a nonzero fracture probability of $P_{r}(0, i)$. This $P_{r}(0, i)$ is then compared with a random number $P^{*}(0, i)$ generated uniformly within the interval of $[0,1]$. If $P_{r}(0, i)>P^{*}(0, i)$, the $i$ th fiber breaks irreversibly and the borne load is redistributed among all other surviving fibers in equal amount. From this point, the broken fiber remains broken and does not contribute to the dynamics. At the next time step $(t=1)$, different rupture probabilities are calculated for the intact fibers based on the new local stress profiles $\sigma(1, i)$. The model keeps evolving at each time by comparing the two probabilities, $P_{r}(t, i)$ and $P^{*}(t, i)$. The present authors adopt essentially the same time evolution rule in a fiber bundle model with a deterministic rupture rule (i.e., at $T=0$ ) and find creeplike time evolution [8]. Here we extend this model to investigate the effect of stochasticity, the amplitude of which may be proportional to temperature.

Equation (1) suggests that $P_{r}$ increases with temperature $T$ and the local stress $\sigma(t, i)$. Then the nature of breakdown of the system will be determined by the interplay between the local stress profile, the individual strength of the fibers, and the temperature. In the present study we concentrate on the mean-field model in which $\sigma(t, i)$ are uniform (independent of $i$ ) as a result of democratic load redistribution.

The stress threshold of each fiber, $\sigma_{\text {th }}(i)$, is a random quantity to be sampled from a certain probability distribution, $\rho\left(\sigma_{\text {th }}\right)$. Unless otherwise indicated, we adopt a uniform distribution with the mean of $1 / 2$ and the half-width of $\delta$ :

$$
\rho\left(\sigma_{\text {th }}\right)= \begin{cases}1 / 2 \delta, & \left(1 / 2-\delta<\sigma_{\text {th }}<1 / 2+\delta\right) \\ 0 & \text { (otherwise) } .\end{cases}
$$

\section{NUMERICAL RESULTS}

Numerical results are produced for the mean-field model with system sizes ranging between $10^{3}$ and $10^{5}$. To compute average values of physical quantities such as creep lifetime, $10^{4}-10^{5}$ configurations are sampled at each system size. Here we have mainly studied the behavior of creep lifetime as temperature, applied stress, and extent of disorder is tuned. Additionally, a series of dynamical transitions observed during the creep failure will be discussed in detail.

\section{A. Distribution of creep lifetime}

In this section we discuss the distributions of creep lifetime in the parameter space of $\sigma-T$ to understand whether any extreme statistics is associated with it. Hereafter the creep lifetime is denoted as $\tau_{c}$, which varies from sample to sample. To compute the distributions of creep lifetime, $10^{5}$ samples are computed for the system size of $10^{3}$. Figure 1 shows that the peak of the distribution shifts to lower values as either of the parameters, temperature or applied stress, is increased. This happens as the probability of rupture increases with increasing $\sigma$ or $T$. A simultaneous increment in both parameters shifts the peak even faster.

The distribution of creep lifetime develops a tail, suggesting the existence of extreme statistics. As shown in Figs. 2(a) and 2(d), a closer look at the distribution functions reveals that they are well fitted with the log-normal distribution given 

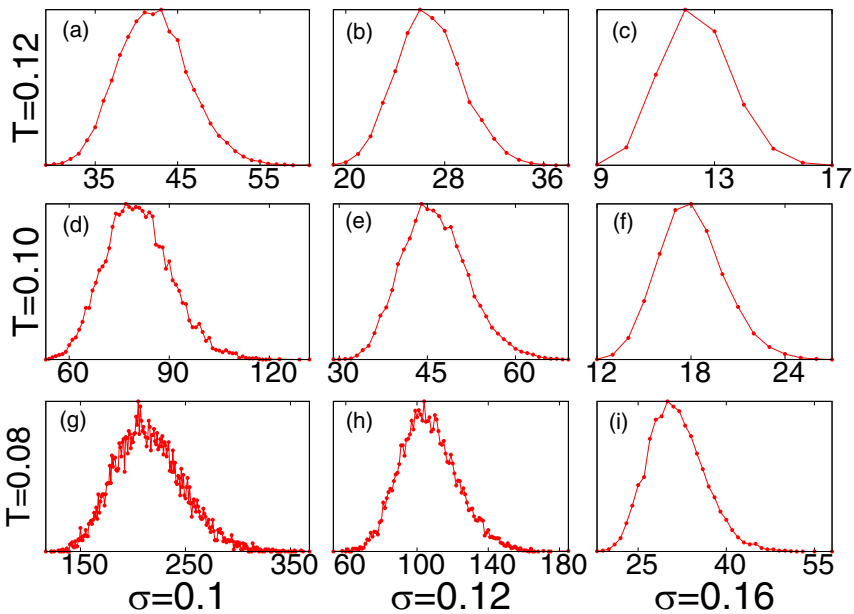

FIG. 1. Distribution of creep lifetime for several parameter values of $(\sigma, T)$. The average of the distribution shifts to lower value if either $\sigma$ or $T$ increases. $\delta$ is kept constant at 0.5 .

below:

$$
P\left(\tau_{c}\right)=\frac{1}{\tau_{c} v \sqrt{2 \pi}} \exp \left[-\frac{\left(\ln \tau_{c}-\mu\right)^{2}}{2 v^{2}}\right],
$$

where $v$ is the variance and $\mu$ is the mean of $\log \tau_{c}$. The values of $(\mu, v)$ are shown in the legends of individual panels of Figs. 2(a) and 2(d). They depend on the applied stress and the temperature as shown in Figs. 2(b), 2(c), 2(e), and 2(f). We observe that the mean value decreases gradually as either the temperature or the applied stress increases. On the other hand, the variance is insensitive to the applied stress but decreases with increasing temperature.
The lifetime distributions are also compared with the threeparameter Weibull distribution, which looks very similar to the log-normal distribution but still can be discriminated [24]. The comparison is shown in Fig. 2(g) for $T=0.08$ and $\sigma=$ 0.14. Apparently, the log-normal distribution fits the simulation result better, particularly at the tails. The distribution remains the same at any temperature and stress, as well as in the limit $T \rightarrow 0$ [9] (and hence applied stress $\geqslant$ critical stress), where the fluctuation in creep time arises from the fluctuation of critical stress for different configurations.

At a constant temperature and varying applied stress, we observe the following scaling:

$$
P\left(\tau_{c}\right) \simeq \sigma^{\beta} \Phi\left(\tau_{c} \sigma^{\beta}\right),
$$

with $\beta=4.0$. The scaling is demonstrated in Fig. 3(a) for different applied stress but at a constant temperature $T=0.08$. The inset of the same figure shows the unscaled behavior. The above scaling also tells that, at constant temperature $T$, the average lifetime decreases with the applied stress in a scale-free manner with a temperature-dependent exponent, $\beta$ :

$$
\left\langle\tau_{c}\right\rangle \sim \sigma^{-\beta(T)} \text {. }
$$

The behavior is examined numerically and shown in Fig. 3(b). The exponent $\beta$ is a decreasing function of temperature. This is understandable since the probability at high temperature is high enough to create abrupt failure independent of how much stress is applied on the bundle. In this limit, the creep time will be very small, and the affect of applied stress on creep time will be absent.

\section{B. Transitions in abruptness}

In this section, we have studied the abruptness of failure. Denoting the number of unbroken fibers at time $t$ as $L_{t}$, we
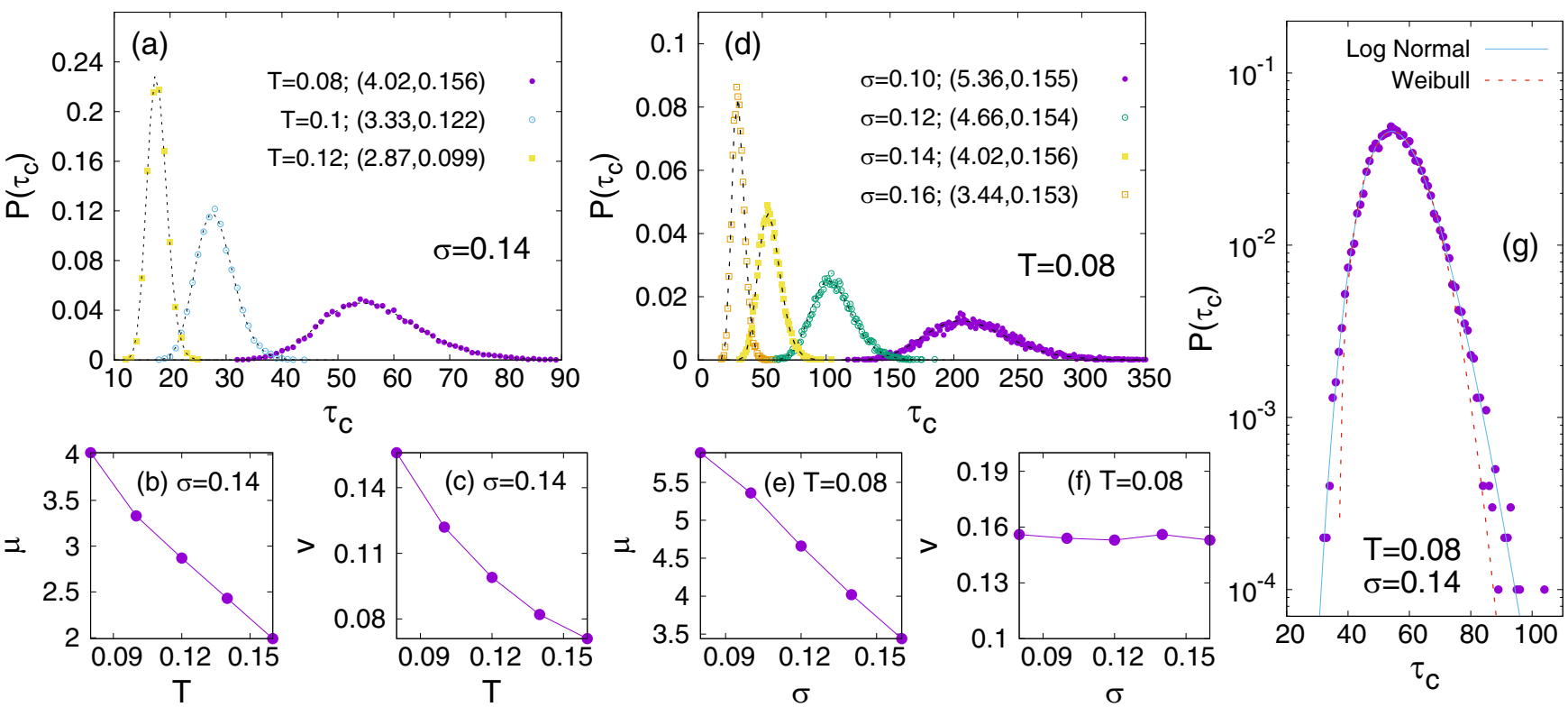

FIG. 2. (a) Distribution of creep lifetime at applied stress $\sigma=0.14$ and temperature $T=0.08,0.1$, and 0.12 . The mean and the variance of $\log \tau_{c}$ depend on temperature $T$ as shown in (b) and (c), respectively. (d) Distribution of creep lifetime at temperature $T=0.08$ and applied stress $\sigma=0.1,0.12,0.14$, and 0.16 . The mean and the variance of $\log \tau_{c}$ depend on the stress as shown in (e) and (f), respectively. (g) Lifetime distribution at $T=0.08, \sigma=0.14$ are fitted with log-normal distribution and three-parameter Weibull distribution. The comparison shows a better fit for log-normal distribution. $\delta$ is kept constant at 0.5 . 

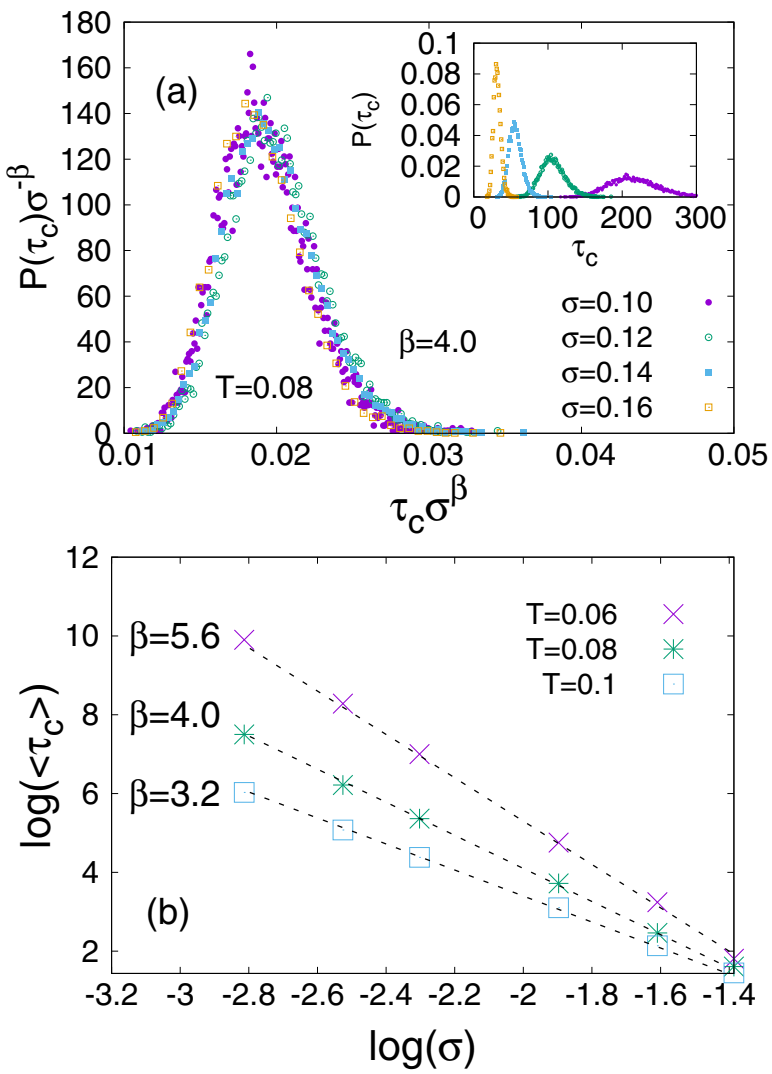

FIG. 3. (a) At a constant $T(=0.08)$, as we vary the applied stress $\sigma$ we observe the following scaling: $\sigma^{-\beta} P\left(\tau_{c}\right) \sim \Phi\left(\tau_{c} \sigma^{\beta}\right)$, where $\beta=4.0$. The inset shows the unscaled behavior. (b) Variation of average creep lifetime $\left\langle\tau_{c}\right\rangle$ with applied stress for three different temperatures. We keep $\delta$ fixed at 0.5 .

define the decreasing rate of fibers as $s(t)=L_{t}-L_{t+1}$. Then the time average of $s(t)$ is computed for each sample from $t=0$ to the time of creep failure, $\tau_{c}$. This time-averaged value of $s(t)$ is denoted by $s$. For instance, $s=L$ if $\tau_{c}=1$, and $s=L / 2$ if $\tau_{c}=2$, and so forth. (Here $L=L_{0}$.) Indeed, one can easily confirm that $s=L / \tau_{c}$. Larger $s$ thus implies that the breakdown is more abrupt. Since this $s$ varies from sample to sample, the ensemble average, which is denoted by $\langle s\rangle$, is taken over $10^{4}$ configurations. Note that $\langle s\rangle / L=$ $\left\langle\tau_{c}^{-1}\right\rangle$. This is interpreted as the average breakdown rate, and we regard this quantity as an order parameter for the abruptness.

Figure 4 shows the behavior of $\langle s\rangle / L$. The applied stress $\sigma$ is varied from 0.1 to 0.4 , while the extent of disorder $\delta$ ranges between 0.1 and 0.3 . At high temperatures, the total bundle breaks in a single time step, i.e., $\langle s\rangle / L=1$. As temperature decreases, $\langle s\rangle / L$ decreases abruptly at a certain temperature, and then similar drops occur repeatedly with smaller amplitudes. Specifically, the first jump is from 1 to $1 / 2$, and the second jump is from $1 / 2$ to $1 / 3$, and so on. The height of the $n$th jump, denoted by $\Delta h_{n}$, is

$$
\Delta h_{n} \simeq \frac{1}{n(n+1)} .
$$

This is understandable if one recalls that $\langle s\rangle / L=\left\langle\tau_{c}\right\rangle$ and that $\tau_{c}$ increases from $n$ to $n+1$ for the $n$th jump. Figure 4
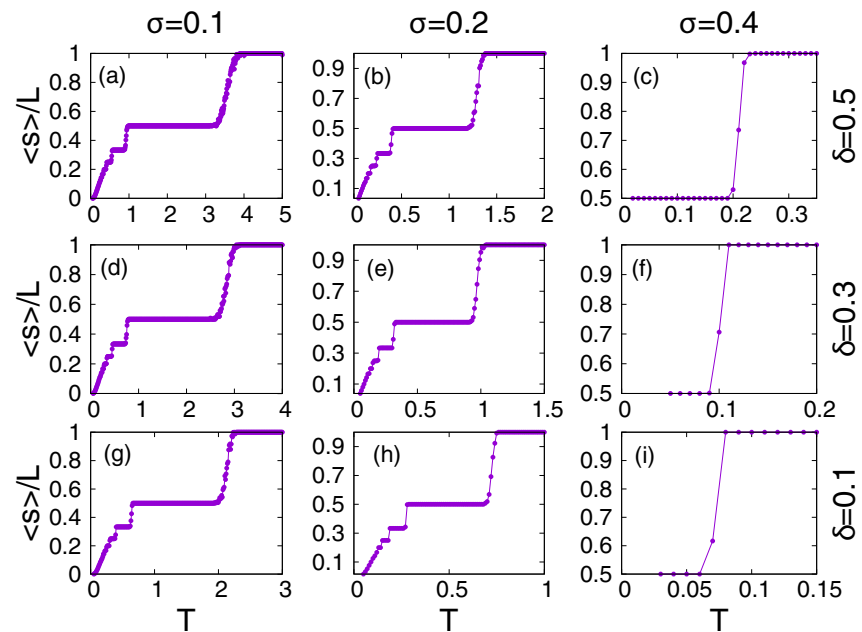

FIG. 4. The average breakdown rate $\langle s\rangle / L$ is shown as a function of temperature at several values for the extent of disorder (horizontally) and external stress (vertically). The behavior is characterized by several discontinuous jumps. The number and the temperatures of these jumps depend on the stress $\sigma$, while the extent of disorder $\delta$ affects the temperatures only.

also shows that the jump height increases with stress $\sigma$. For example, there are a number of jumps with decreasing jump heights for $\sigma=0.1$, whereas for $\sigma=0.4$ there is a single jump from 1 to $1 / 2$. This is because the system ruptures within only one or two time steps after a few large avalanches. This behavior remains almost unaltered with increasing the extent of disorder, whereas the jump temperatures are affected.

To study these jumps more closely, we have carried out the finite-size scaling. Figure 5 shows the behavior of $\langle s\rangle / L$ at several system sizes ranging from $L=5 \times 10^{2}$ to $5 \times 10^{4}$. The scaling form is given as follows:

$$
\langle s\rangle / L \sim \Psi\left[\left(T-T_{n}^{c}\right) L^{\alpha}\right]
$$



FIG. 5. Variation of average avalanche size $\langle s\rangle / L$ with increasing temperature $T$ at applied stress $\sigma=0.15$ and the extent of disorder $\delta=0.5$. The inset shows the system size scaling for the first jump, $\langle s\rangle / L \sim \Psi\left[\left(T-T_{1}^{c}\right) L^{\alpha}\right]$, where $\alpha=0.45$ and $T_{1}^{c}=2.12$. 

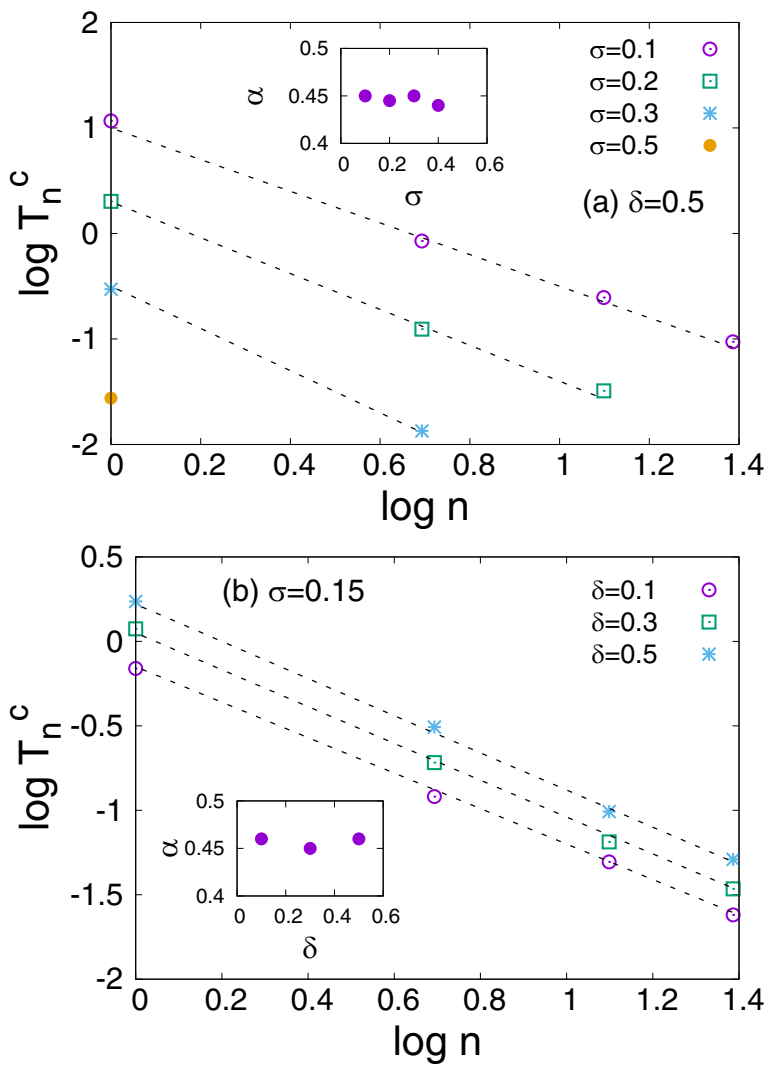

FIG. 6. Variation of the critical temperature $T_{n}^{c}$ with the jump number $n$ for different $\sigma$ at $\delta=0.5$ (a) and for different $\delta$ at $\sigma=0.15$ (b). The inset shows the variation of $\alpha$ at $n=1$. The jump temperatures $T_{n}^{c}$ are observed to decrease in a power-law fashion with the exponent depending on stress and the extent of disorder: (a) 2.0 $(\sigma=0.3), 1.7(\sigma=0.2)$, and $1.5(\sigma=0.1)$; (b) $1.05(\delta=0.1), 1.09$ $(\delta=0.3)$, and $1.1(\delta=0.5)$.

where $T_{n}^{c}$ is the temperature at the $n$th jump, and the exponent $\alpha$ is a constant. The inset of Fig. 5 shows the finite-size scaling for $n=1$ with $T_{1}^{c}=2.12$, where the scaling exponent $\alpha$ is approximately 0.45 . This value is irrespective of the stress $\sigma$ and the extent of disorder $\delta$, as shown in the insets of Fig. 6. Here the exponent is estimated for the first jump $(n=1)$, but we confirm that this behavior is also independent of the jump number $n$.

The jump temperatures $T_{n}^{c}$ and the interval between two consecutive jumps, $T_{n+1}^{c}-T_{n}^{c}$, decrease with $n$. Figure 6 shows the variation of $T_{n}^{c}$ with $n$ for different values of stress $\sigma$ (panel a) and the extent of disorder $\delta$ (panel b). Both panels indicate the decrease in $T_{n}^{c}$ with $n$ in a power-law fashion. The exponent depends on the stress and the extent of disorder. The transition temperatures $T_{n}^{c}$ decrease with increasing applied stress, while they increase with the extent of disorder.

Figure 7 shows the variation of $T_{1}^{c}$ and $T_{2}^{c}$ with the applied stress $\sigma$ and the extent of disorder $\delta$. The two planes correspond to the first $\left(T_{1}^{c}\right)$ and second $\left(T_{2}^{c}\right)$ transitions, respectively. The region above the first $(n=1)$ plane corresponds to the instantaneous failure when the system breaks in a single time step, resulting in the smallest creep lifetime $\left(\tau_{c}=1\right)$. In this region, the distribution of $\tau_{c}$ is a $\delta$ function at 1 .

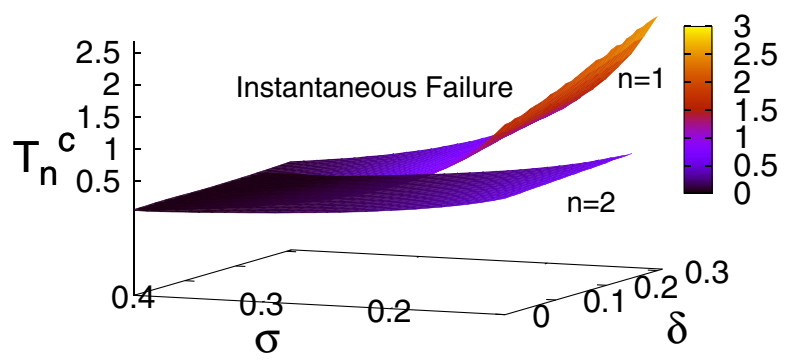

FIG. 7. Variation of the critical temperature $T_{n}^{c}$ with the applied stress $\sigma$ and the extent of disorder $\delta$. The variation is shown for the first $(n=1)$ and second $(n=2)$ transition.

\section{UNIVERSALITY}

We have carried out numerical simulations using the uniform threshold distribution for fiber strength. To see the robustness of the results, two other functions are adopted as the threshold distribution: a Gaussian distribution and a power-law distribution. The exponent for the power-law distribution is -1 and spans the range of $\left[10^{-\eta}, 10^{\eta}\right]$, with $\eta$ representing the extent of the disorder. We have confirmed that the results are mostly independent of the choice of threshold distributions. See Appendix for further details.

\section{DISCUSSION}

In the present work, the effect of temperature in creep failure is probed by a fiber bundle model with a probabilistic algorithm. As a result of the stochasticity, the model can fail even if the applied stress is below the critical value when $T>0$. The dynamical properties of the model, including the exponents for power-law behaviors, are comparable to those of real materials. The model exhibits a large scatter in the creep lifetime, which can be described by a log-normal distribution with the temperature-dependent mean and variance. The mean lifetime depends on the applied stress in a scale-free manner.

For practical purposes, the average lifetime alone does not provide us with sufficient information on creep failure since the lifetime distribution is skewed [25]. Both the log-normal and the Weibull distributions are observed for the lifetime of some alloys [26,27]. The Weibull distribution was also found for STS304 stainless steel [28]. In our study, the creep lifetime distribution is fitted more convincingly with the log-normal distribution than the Weibull distribution. Another major difference is, in our case the Weibull distribution contains three parameters, while the above experimental creep lifetimes are fitted well with the two-parameter Weibull distribution. In addition, the stress dependence of the lifetime in our model agrees well with experimental behaviors [29-31]. In [29], the authors showed a scale-free decrease of creep lifetime for a MarBN steel variant at elevated temperatures. Similar to our numerical results, the exponent of the power law was observed to decrease (from 19.77 to 13.02) as the temperature is increased $(625-675 \mathrm{~K})$. Reference [30] explored the powerlaw behavior of applied stress with creep time for Grade 22 gold and observed a nice fitting there. The power-law exponent here also follows an increasing trend with decreas- 

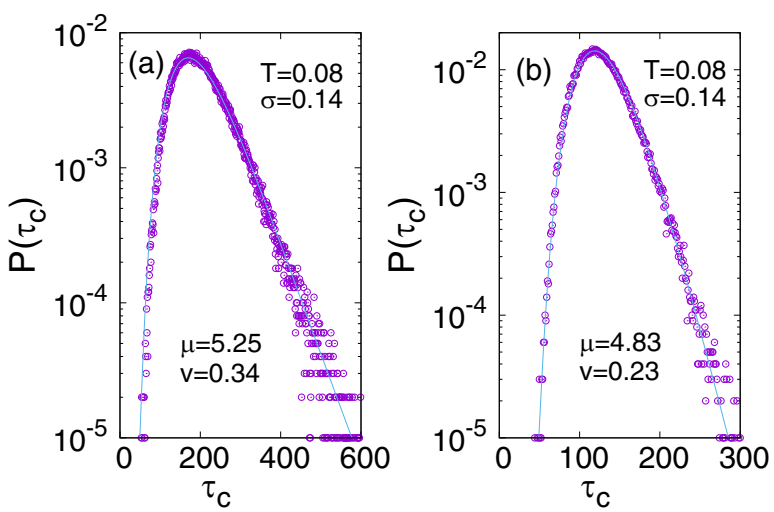

FIG. 8. Distribution of the creep lifetime is fitted with the lognormal distribution. The threshold distribution is (a) power law and (b) Gaussian, respectively.

ing temperature (goes from 4 to 16 when temperature is increased from 723 to $923 \mathrm{~K}$ ). The same has been done for various kinds of steels in [31]. Apart from engineering, the stress dependence, discussed above, is also observed in medical science during the failure of human achilles tendons under creep and cyclic loading [32]. In addition to the stress dependence, an exponential increase in creep lifetime has been observed in Refs. [30] and [31] with temperature. Such increments with temperature have already been observed in fiber bundle creep lifetime in an early study [22]. Overall, the present numerical results are mostly consistent with experimental results on materials [25-29].

We have also observed many jumps in the average breakdown frequency, $\langle s\rangle / L$. Since these jumps obey a finite-size scaling, they may be an analog of phase transitions. However, the average breakdown frequency involves the dynamic property (i.e., breakdown) and therefore a nonequilibrium quantity. Thus, these jumps may be nonequilibrium phase transitions or dynamical transitions, to which the conventional theory for phase transitions does not apply. The correlation length is also an important quantity from the viewpoint of phase transitions; we cannot define any length scale here as there are no spatial degrees of freedom. To this end, we need to analyze the local stress redistribution model.
Understanding of creep behavior is a fundamental problem in materials science and engineering. Prior knowledge on the creep lifetime for a particular material reduces the threat of sudden catastrophic failure and increases predictability in the failure process. At the same time, sufficient knowledge of creep dynamics makes us aware if the system is approaching the global failure. In this paper, we have presented a detailed study of the creep dynamics in a disordered system. Our next step is to explore the model with other modifications, such as the inclusion of stress concentration around a broken fiber. Application of time-dependent loading allows us to explore the possibility of fatigue within the model.

\section{APPENDIX A: OTHER THRESHOLD DISTRIBUTIONS}

To check the robustness of our numerical result, we have repeated some of the numerical results with other distributions. For this purpose, we have adopted two different distributions:

(i) A power-law distribution ranging from $10^{-\eta}$ to $10^{\eta}$ :

$$
\rho\left(\sigma_{\text {th }}\right)= \begin{cases}\sigma_{\text {th }}^{-1}, & \left(10^{-\eta}<\sigma_{\text {th }}<10^{\eta}\right) \\ 0 & (\text { otherwise }) .\end{cases}
$$

(ii) A Gaussian distribution around a mean $\mu_{g}=0.5$ and variance $v_{g}=0.5$ :

$$
\rho\left(\sigma_{\mathrm{th}}\right)=\frac{1}{\sqrt{2 \pi v_{g}^{2}}} \exp \left[-\frac{\left(\sigma_{t h}-\mu_{g}\right)^{2}}{2 v_{g}^{2}}\right] .
$$

For the power-law distribution, $\eta$ measures the extent of disorder. On the other hand, the amount of disorder for the Gaussian distribution is determined by $\mu_{g}$ and $v_{g}$. For all the numerical results, shown below, we have kept these parameters fixed at $\eta=0.3, \mu_{g}=0.5$, and $v_{g}=0.5$, respectively.

The distribution of the creep lifetime is fitted with the log-normal distribution for both the threshold distributions [see Figs. 8(a) and 8(b), respectively]. We have also studied the stress dependence of the average creep lifetime at several values of temperatures: $0.06,0.08$, and 0.1 . We retain the
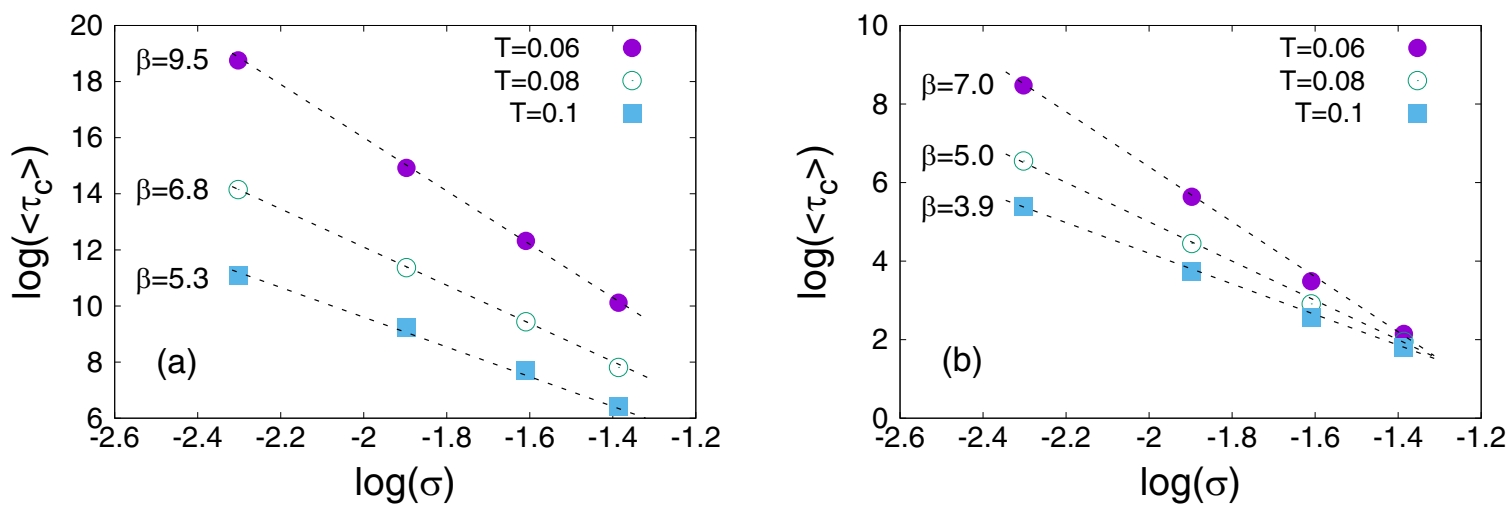

FIG. 9. Variation of average creep lifetime $\left\langle\tau_{c}\right\rangle$ with applied stress for three different temperatures: $T=0.06,0.08$, and 0.1 . The extent of disorder for (a) power-law and (b) Gaussian distribution is kept constant. 

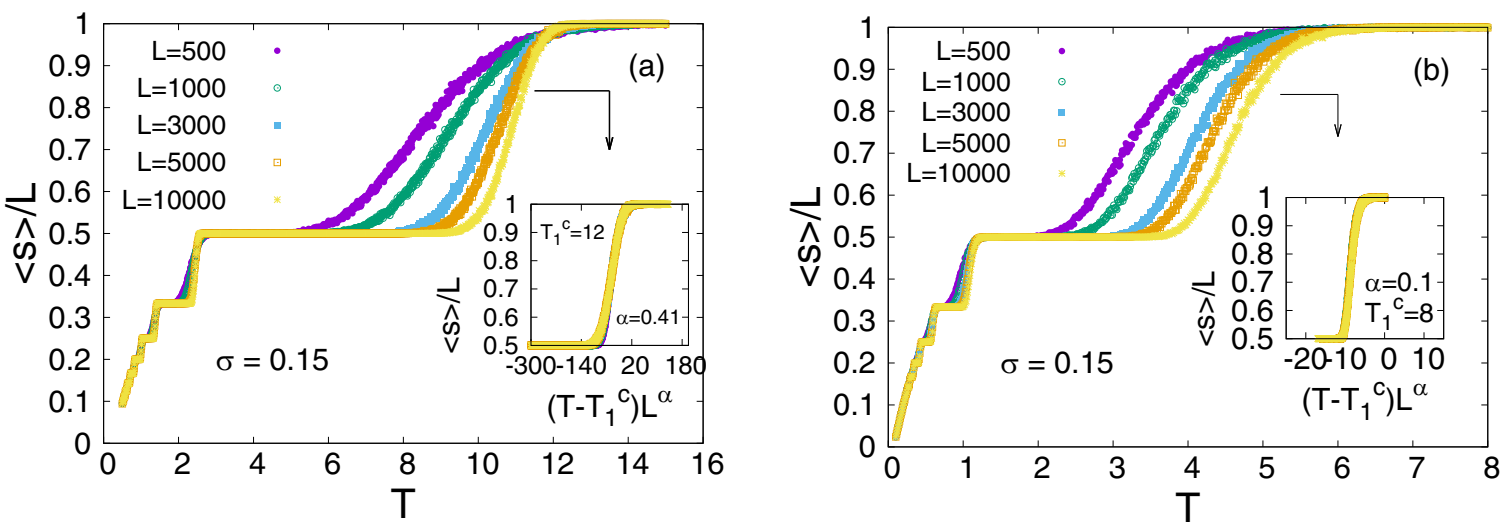

FIG. 10. Variation of average avalanche size $\langle s\rangle / L$ with increasing temperature $T$ at applied stress $\sigma=0.15$ and a certain degree of disorder for (a) power-law and (b) Gaussian distribution. The inset shows the system size scaling: $\langle s\rangle / L \sim \Psi\left[\left(T-T_{1}^{c}\right) L^{\alpha}\right]$.

power-law behaviors,

$$
\left\langle\tau_{c}\right\rangle \sim \sigma^{-\beta(T)},
$$

where the exponent $\beta$ is a decreasing function of the temperature in both cases. See Figs. 9(a) and 9(b).

Figures 10(a) and 10(b) show the dynamical transitions associated with the abruptness during the failure for power-law and Gaussian distribution, respectively. We observe that the average size of avalanches exhibits a number of transitions by varying the temperature. Thus, the transitions are not affected by the choice of threshold distribution, at least for the uniform, power-law, and Gaussian distributions:

$$
\langle s\rangle / L \sim \Psi\left[\left(T-T_{n}^{c}\right) L^{\alpha}\right] .
$$

We observe $\alpha=0.41$ for power-law and 0.1 for the Gaussian distribution. The transition temperatures ( $T^{c}$ values) are expected to have higher values here, as there will be some fibers of very high threshold values (though with smaller probabilities) and the temperature has to be higher to achieve instantaneous failure. Here, the temperature corresponding to first transition is observed to be $T_{1}^{c}=12$ and $T_{1}^{c}=8$, respectively.
[1] B. R. Lawn, Fracture of Brittle Solids (Cambridge University Press, Cambridge, England, 1993).

[2] T. Wong and P. Baud, J. Struct. Geol. 44, 25 (2012).

[3] I. G. Main, Geophys. J. Int. 142, 151 (2000).

[4] B. Voight, Science 243, 200 (1989).

[5] A. Hansen, P. C. Hemmer, and S. Pradhan, The Fiber Bundle Model: Modeling Failure in Materials (Wiley-VCH, Weinheim, 2015).

[6] F. T. Pierce, J. Text. Ind. 17, T355 (1926).

[7] H. E. Daniels, Proc. R. Soc. London, Ser. A 183, 405 (1945).

[8] S. Roy and T. Hatano, Phys. Rev. E 97, 062149 (2018).

[9] S. Roy, S. Biswas, and P. Ray, Phys. Rev. Res. 1, 033047 (2019).

[10] S. Roux, Phys. Rev. E 62, 6164 (2000).

[11] S. Ciliberto, A. Guarino, and R. Scorretti, Physica D 158, 83 (2001).

[12] R. Scorretti, S. Ciliberto, and A. Guarino, Europhys. Lett. 55, 626 (2001).

[13] A. Politi, S. Ciliberto, and R. Scorretti, Phys. Rev. E 66, 026107 (2002).

[14] A. Saichev and D. Sornette, Phys. Rev. E 71, 016608 (2005).

[15] N. Yoshioka, F. Kun, and N. Ito, Phys. Rev. E 82, 055102(R) (2010).

[16] N. Yoshioka, F. Kun, and N. Ito, Europhys. Lett. 97, 26006 (2012).

[17] R. C. Hidalgo, F. Kun, and H. J. Herrmann, Phys. Rev. E 65, 032502 (2002).
[18] F. Kun, Y. Moreno, R. C. Hidalgo, and H. J. Herrmann, Europhys. Lett. 63, 347 (2003).

[19] E. A. Jagla, Phys. Rev. E 83, 046119 (2011).

[20] H. Nechad, A. Helmstetter, R. El. Guerjouma, and D. Sornette, J. Mech. Phys. Solids 53, 1099 (2005).

[21] Z. Danku and F. Kun, Sci. Rep. 3, 2688 (2013).

[22] S. Pradhan and B. K. Chakrabarti, Phys. Rev. E 67, 046124 (2003).

[23] S. Pradhan, A. K. Chandra, and B. K. Chakrabarti, Phys. Rev. E 88, 012123 (2013)

[24] R. Dumonceaux and C. E. Antle, Technometrics 15, 923 (1973).

[25] L. Xing, J. Zhang, F. Shen, and W. Feng, Int. J. Press. Vessels Piping 83, 730 (2006).

[26] M. Evans, J. Mater. Proc. Tech. 54, 171 (1995).

[27] M. Evans and A. R. Ward, Mater. Sci. Technol. 16, 1149 (2000).

[28] S. J. Kim, Y. S. Kong, Y. J. Noh, and W. K. Kim, Mater. Sci. Eng. A 483-484, 529 (2008).

[29] A. Benaarbia, X. Xu, W. Sun, A. A. Becker, and M. A. E. Jepson, Mater. Sci. Eng. A 734, 491 (2018).

[30] Z. Abdallah, V. Gray, M. Whittaker, and K. Perkins, Materials (Basel) 7, 3371 (2014).

[31] S. S. Manson and A. M. Haferd, A Linear Time-Temperature Relation for Extrapolation of Creep and Stress-Rupture Data, National Advisory Committee for Aeronautics (NACA) (Washington, DC, 1953).

[32] T. A. L. Wern, D. P. Lindsey, G. S. Beaupre, and D. R. Carter, Ann. Biomed. Eng. 31, 710 (2003). 